\title{
Robustness modelling and assessment of interdependent smart grids
}

\author{
Ferdinand von Tüllenburg \\ From The 7th DACH+ Conference on Energy Informatics \\ Oldenburg, Germany. 11-12 October 2018
}

Correspondence: ferdinand. tuellenburg@salzburgresearch.at Salzburg Research

Forschungsgesellschaft mbH, Salzburg, Austria

\begin{abstract}
Future smart grids heavily rely on collaboration of two interdependent subsystems: the information and communication (ICT) system and the electrical system itself. The past has shown that malfunctions in one of the systems can propagate into the other system leading to widespread and heavy outages of power supply. Within this PhD project, a methodology for modeling, assessing and quantifying the Smart Grids' robustness against occurrence of important unpredictable events is developed. Special attention is paid to the interdependencies between ICT and electrical subsystem and a particular novelty to existing approaches is the robustness quantification based on the current state of both subsystems allowing constant evaluation.
\end{abstract}

Keywords: Smart grid, Robustness, Modeling, Interdependent systems

\section{Motivation}

The information- and communication (ICT) subsystem and the electrical subsystem of modern power systems are interdependent. This means both subsystems have direct or indirect influence on each other because the ICT subsystem provides the necessary communication capabilities, while the electrical subsystem provides the necessary electrical power to ICT equipment. However, past has shown that those interdependencies may pave the way for failure cascades between both subsystems leading to uncontrolled behaviour and total loss of control (Buldyrev et al., 2010). For example, in September 2003, a ground fault of a transmission power line propagated also into the ICT system causing power losses in central communication nodes. The result has been degraded functionality of the operators' supervisory control and data acquisition (SCADA) system and finally complete loss of control. At the end, the failure cascade led to a large scale blackout in Italy with about 56 million affected people (UCTE, 2007). One particular challenge of handling these interdependencies is that they are subject to continuous change due to reconfigurations and altering conditions. For instance, abrupt shifts of power consumption or production or topological changes due to component failures. Thus, in this work, a methodology is developed enabling grid operators to (1) identify interdependencies between the ICT and electrical subsystems during run time under consideration of dynamically changing states and configurations, and (2) to assess the relevance of those

(C) The Author(s). 2018 Open Access This article is distributed under the terms of the Creative Commons Attribution 4.0 International License (http://creativecommons.org/licenses/by/4.0/), which permits unrestricted use, distribution, and reproduction in any medium, provided you give appropriate credit to the original author(s) and the source, provide a link to the Creative Commons license, and indicate if changes were made. 
interdependencies according to the overall system robustness. In view of the fact that faults may easily propagate between both subsystems, continuous assessment of Smart Grid robustness is crucial for mitigation of cascading failures between both subsystems.

\section{Research questions}

The top-level research question of this work is: How can machine learning methods applied to assess (typecast and quantify) interdependencies of distribution systems and corresponding ICT systems during operation in order to derive statements to the overall system robustness?

In context of electrical systems, robustness is usually defined as the capability of the electrical system to deliver electrical power supply within expected quality even in the presence of active failures or suddenly materializing incidents. The envisaged methodology aims at evaluating the robustness of the electrical system with particular focus on the possible risk caused by interdependencies. In order to address the overall target, this $\mathrm{PhD}$ project is subdivided into the following research questions:

RQ1: How is the robustness of a distribution system influenced by incidents happening in the electrical subsystem or the ICT subsystem?

The outcome of this question is a model showing how different types of interdependencies (cyber, physical, geographical (Ouyang, 2014)) and particular classes of incidents may affect the power quality of a distribution system after a cascade of failures.

RQ2: How can robustness of the overall system be quantified? Given the outcomes of RQ1 a model is developed, how the effects on system robustness can be quantified by expressing the impact incidents have on the system robustness. This quantification comprises also properties of particular systems such as node degrees and component capacities.

RQ3: How to apply machine learning capable of autonomously evaluate overall system robustness? The answer of RQ3 will contain what components the assessment system might comprise of. Which of the components are already available and which are required to be developed in order to build the assessment system. Furthermore, it is required to define how functional (e.g. detecting and assessing interdependencies under constantly changing conditions and configurations) and non-functional requirements (e.g. comparatively short computation time to allow online evaluation).

\section{Status of the PhD-project}

The project has just started. Currently most work concentrates on RQ1 by analysing and classifying possible incidents in ICT of power systems and the electrical system itself including cascading and propagating effects. Finalization of the project is planned by the end of 2020. The PhD project is going to be supervised by Prof. Dr. Sven Tomforde (University of Passau) and Prof. Dr. Andreas Berl (Deggendorf Institute of Technology).

\section{Related work}

The analysis of interdependencies and cascading failures between separate systems and in particular in context of electrical grids have been investigated in research extensively (Buldyrev et al., 2010; Ouyang, 2014; Wäfler \& Heegaard, 2013). However, these approaches operate mainly on a qualitative analysis not trying to quantify the effects 
those interdependencies might have on the overall system output, if one or more components get unavailable suddenly.

On the other hand, also several approaches are proposed for definition, analysis and quantification of robustness in electrical systems (Crucitti et al., 2004; Kinney et al., 2005; Motter \& Lai, 2002; Lai et al., 2004) only to name a few. Those approaches, however, are not designed for fast evaluation, which would be required in terms of future smart grid environments with rapidly changing conditions and constantly changing system configurations.

\section{Methodical approach towards robustness assessment}

In the first step, information of the currently active topology is retrieved from the energy management system (EMS) and network management system (NMS). The information from both sources is mapped onto each other in order to derive physical and cyber dependencies between both subsystems. This means in particular, with respect to physical dependencies it is derived which parts of the electrical system are delivering power to which parts of the ICT system. Regarding cyber dependencies it is derived which parts of the ICT system are involved to provide necessary data communication capabilities to the corresponding grid components. In the next step, the interdependency description is subject to expert analysis. In this step, in particular certain derived interdependencies might be removed as, e.g., independent power supplies (UPS) or redundant ICT components provide protection against physical or informational dependencies.

From that information an interdependency graph can be constructed containing for each component in one of the subsystems, from which components in the other subsystem it is dependent. An exemplary distribution system comprising of an ICT subsystem and an electrical subsystem is depicted in (Fig. 1). The electrical system consists of three transformer stations $\left(T_{1}-T_{3}\right)$, connected to a system of power lines, line switches $\left(\mathrm{A}_{1}-\mathrm{A}_{3}\right)$ and bus bars where power lines are interconnected. At the ICT side, the power system is equipped with several intelligent electronic devices (IEDs) denoted with $\mathrm{M}_{0}-\mathrm{M}_{9}$, which are necessary for proper power grid control. In particular, IEDs are connected to the grid control systems by utilization of an ICT network comprising of four switches connected in a ring topology $\left(S_{1}-S_{4}\right)$. This network



Fig. 1 Power system comprising of ICT and electrical subsystem 
provides connection for the IEDs and the central control stations $\mathrm{C}_{1}$ and $\mathrm{C}_{2}$. Data transfer links are depicted as green lines, where solid lines represent active links and dashed lines represent inactive links. Finally, in the current grid configuration three supply regions $\left(R_{1}-R_{3}\right)$ are depicted, where $R_{1}$ is supplied by transformer $T_{3}$, region $R_{2}$ is supplied by $T_{2}$ and region $R_{1}$ is supplied by transformer $T_{1}$. The interdependency graph for component $\mathrm{C}_{2}$ (a control system) is shown in (Fig. 2). The graph shows for component $C_{2}$ (a control system) which dependencies exist to other components in order to operate properly. The component denotations are taken from (Fig. 1). Dashed lines represent dependencies within the same subsystem, for instance, the dependency of $\mathrm{C}_{2}$ on proper functionality of Switch $\mathrm{S}_{3}$. Solid lines visualizing interdependencies into the other subsystem. In the given example, switch $\mathrm{S}_{3}$ has an interdependency on power supply provided by transformer $\mathrm{T}_{2}$. It gets apparent from the figure that all of the corresponding components are interdependent with the electrical system finally.

The resulting information about system structure and interdependencies is fed into a cascading failure simulation system. This provides a simulation model with the necessary data basis to derive how extracted physical, informational and geographical interdependencies affect the both subsystems. For incidents, appearing at one component of either subsystem it can be evaluated how they spread into the other subsystem (i. e. hit components in the other system). For both subsystems already (from literature) available simulation models for cascading failures might be applied. At this state, the simulation model comprises of static information valid for given configurations of the sub networks. However, for the robustness analysis important information on type and number of dependencies between the components or nodes of the subsystem can be derived. Within this model different configurations of the subsystems, resulting in different information flows and power flows in the subsystem can be evaluated in order to derive changes of interdependencies (in particular physical and informational) between the subsystems.

In the next steps, the model will be complemented with dynamic aspects of the smart distribution system. In addition to metrics such as node degrees (which can be derived



Fig. 2 An interdependency graph derived from topological information 
from structural analysis) especially load flows and component capacities provide important measures for evaluating a nodes relevance. The corresponding robustness metric is developed as part of RQ2. By simulating certain load/consumption situations including load flows in the available topologies, the nodes' relevance is evaluated and, thus, the robustness of the overall system can be derived.

By employing realistic load/consumption situations and corresponding system utilization including load flows (gathered, e. g., from historical data), the simulation can be used to train a machine-learning based evaluation system, which can derive system robustness using current subsystem configuration and current load/consumption situations without the need for computationally expensive and time consuming calculations.

\section{Conclusion and outlook}

In this $\mathrm{PhD}$ project, a model for description and assessment of Smart Grid robustness is developed. It is envisioned to quantify the robustness of a Smart Grid using and combining particular models for both, the ICT subsystem and the electrical subsystem of the grid. The main contribution of this project is to allow power grid operators to examine the resilience of the electrical system with respect to failures, which could propagate and cascade between both subsystem induced by unpredicted events. Considered as extensions to commonly used n-1 computations and load-flow analysis the proposed assessment method allows for more precisely estimate the risk of power quality degradations in future Smart Grid deployments.

Abbreviations

EMS: Energy management system; ICT: Information and communication technology; IED: Intelligent electronic device: NMS: Network management system; RQ: Research question; SCADA: Supervisory control and data acquisition system; UPS: Uninterruptable power supply

\section{Funding}

Publication costs for this article were sponsored by the Smart Energy Showcases - Digital Agenda for the Energy Transition (SINTEG) programme.

The work in this paper is partly financed by the project Callia part of the ERA-Net Smart Grids Plus initiative and carried out in behalf of the Austrian Federal Ministry for Transport, Innovation and Technology (BMVIT) with support from the European Union's Horizon 2020 research and innovation programme.

\section{About this supplement}

This article has been published as part of Energy Informatics Volume 1 Supplement 1, 2018: Proceedings of the 7th $\mathrm{DACH}+$ Conference on Energy Informatics. The full contents of the supplement are available online at https:// energyinformatics.springeropen.com/articles/supplements/volume-1-supplement-1.

Authors' contributions

The author has read and approved the final manuscript.

Competing interests

The author declares that he has no competing interests.

\section{Publisher's Note}

Springer Nature remains neutral with regard to jurisdictional claims in published maps and institutional affiliations.

Published: 10 October 2018

References

Buldyrev SV, Parshani R, Paul G, Stanley HE, Havlin S (2010) Catastrophic cascade of failures in interdependent networks. Nature 464(7291):1025-1028

Crucitti P, Latora V, Marchiori M (2004) Model for cascading failures in complex networks. Phys Rev E 69(4):045104

Kinney R, Crucitti P, Albert R, Latora V (2005) Modeling cascading failures in the north American power grid. Eur Phys J B 46(1):101-107

Lai Y-C, Motter AE, Nishikawa T (2004) Attacks and cascades in complex networks. Compl Netw 650:299-310 
Motter AE, Lai Y-C (2002) Cascade-based attacks on complex networks. Phys Rev E 66(6):065102

Ouyang M (2014) Review on modeling and simulation of interdependent critical infrastructure systems. Reliab Eng Syst Saf $121: 43-60$

UCTE (2007) Final report - system disturbance on 4 November 2006

Wäfler J, Heegaard PE (2013) Interdependency modeling in smart grid and the influence of ICT on dependability,

Meeting of the European Network of Universities and Companies in Information and Communication Engineering, pp 185-196

Submit your manuscript to a SpringerOpen ${ }^{\circ}$ journal and benefit from:

- Convenient online submission

- Rigorous peer review

- Open access: articles freely available online

- High visibility within the field

- Retaining the copyright to your article

Submit your next manuscript at $\boldsymbol{\nabla}$ springeropen.com 